\title{
An in vivo dual-luciferase assay system for studying translational recoding in the yeast Saccharomyces cerevisiae
}

\author{
JASON W. HARGER ${ }^{1,2}$ and JONATHAN D. DINMAN ${ }^{2}$ \\ ${ }^{1}$ Department of Molecular Genetics, Microbiology and Immunology, UMDNJ Robert Wood Johnson Medical School, \\ Piscataway, New Jersey 08854, USA \\ ${ }^{2}$ Department of Cell Biology and Molecular Genetics, University of Maryland, College Park, Maryland 20742, USA
}

\begin{abstract}
A new in vivo assay system has been developed to study programmed frameshifting in the yeast Saccharomyces cerevisiae. Frameshift signals are inserted between the Renilla and firefly luciferase reporter genes contained in a yeast expression vector and the two activities are directly measured from cell lysates in one tube. Similar to other bicistronic reporter systems, this one allows the efficient estimation of recoding efficiency by comparison of the normalized activity ratios from each luciferase protein. The assay system has been applied to HIV-1 and L-A directed programmed -1 frameshifting and Ty 1 and Ty3 directed +1 frameshifting. The assay system is amenable to high-throughput screening.
\end{abstract}

Keywords: Virus; ribosome; translation; frameshifting; bicistronic; lysate

\section{INTRODUCTION}

Gone are the days of "one gene one protein" (Beckett and Tatum 1941; Garrod 1902). Cells use numerous molecular mechanisms, for example, gene segment rearrangement and alternative mRNA splicing, to increase the protein-coding capacities of their genomes. The physical constraints imposed by capsid geometries have particularly imposed strict size limits on many viral genomes, which in turn have evolved numerous mechanisms to maximize their informational content. One such solution has been to trick the host cellular translational machinery to misread the genetic code at specific frequencies and at specific locations on viral mRNAs, resulting in the production of two or more distinct proteins. Such translational "recoding" mechanisms can take many forms, including termination codon suppression or misreading, programmed translational frameshifts of one or more nucleotides, and even "hops" over stretches of tens of bases (for review, see Gesteland and Atkins 1996). The genomes of viruses that use programmed ribosomal frameshifting (PRF) are typically organized with the genes encod-

Reprint requests to: Jonathan D. Dinman, Department of Cell Biology and Molecular Genetics, Microbiology Building, Room 2135, University of Maryland, College Park, MD 20742, USA; e-mail: dinman@umd.edu; fax: (301) 314-9489.

Article and publication are at http://www.rnajournal.org/cgi/doi/ 10.1261/rna.5930803. ing enzymatic (e.g., pol) proteins $3^{\prime}$ of, and out of frame with respect to those encoding structural peptides (e.g., Capsid or Gag). The relatively low frequencies of PRF events ( $1 \%-20 \%$; for review, see Farabaugh 1996) result in the synthesis of significantly larger amounts of structural as opposed to enzymatic proteins. Because PRF efficiencies determine ratios of viral structural to enzymatic proteins, it is thought that viral frameshift signals have evolved so as to produce optimum molar ratios of viral proteins for virus particle assembly propagation; indeed, alteration of PRF efficiencies has profound impacts on the propagation of numerous viruses (for review, see Harger et al. 2002). Thus, the development of simple, reliable systems for measuring PRF efficiencies is important for identifying targets and characterizing potential new antiviral therapies.

There have traditionally been two approaches to assaying PRF: direct monitoring of protein products and indirect observation using enzymatic assays. Advantages of enzymatic-based assays of PRF include greater accuracy and reduced costs in terms of both materials and labor. In vivo versus in vitro systems represent a second decision nexus, and again, considerations of accuracy and cost favor in vivo systems. The demonstration that viral PRF is faithfully recapitulated in Saccharomyces cerevisiae (for review, see Farabaugh 1996) makes yeast an ideal platform for the development of high-throughput PRF assay systems. The original yeast-based enzymatic PRF assay systems were based on 
monocistronic lac $Z$ reporter vectors. In these systems, PRF efficiencies are calculated by dividing $\beta$-galactosidase activities produced by cells harboring frameshift test vectors (where $l a c Z$ is situated $3^{\prime}$ of a PRF signal and $\beta$-galactosidase can only be synthesized as a consequence of a PRF event) divided by $\beta$-galactosidase activities measured from cells harboring a 0-frame control plasmid (for review, see Dinman et al. 1998). Though providing a very simple and inexpensive system, complicating factors include the dissimilarity of the reporter mRNAs to viral mRNAs and the need to normalize for cell number/protein concentration, mRNA abundance, and differential translational efficiencies of the PRF reporter and 0-frame reporter mRNAs. Bicistronic reporter systems present a strategy to internally control for these variables. Though a yeast-based lacZ-luciferase bicistronic vector system addresses many of these issues (Stahl et al. 1995), the requirement for two different enzyme assays, high background levels, and the juxtaposition of prokaryotic with eukaryotic genes make this a less than ideal system. Here we report the adaptation of a bicistronic dualluciferase system (Grentzmann et al. 1998) for the measurement of PRF efficiencies in yeast. The simplicity and reproducibility of the assay system coupled with its high degree of sensitivity and specificity overcome the remaining problems

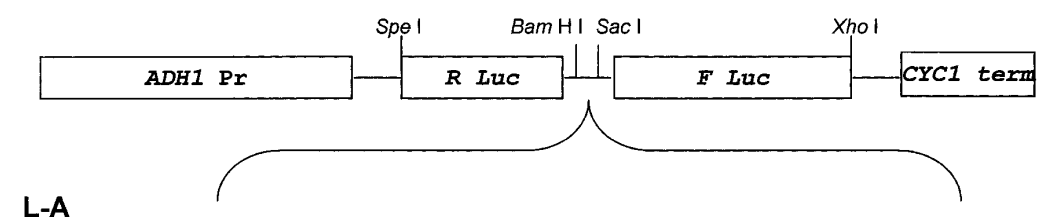

gga tcc act tct agg atc aat geg ggc gaa ctt aag aac tac tgg ggt agt gtg cgt cgt act cag ca gggttta gg agt ggt agg tet tac gat gcc agc tgt aat gcc tac cgg aga acc tac agc tgg cgc tgc cca cga aga gtt gat aga aca gge gga caa tgt ttt agt aga gta aac gta atc gaa cec tca cac gga ccc cgc cet aca agg tac ata ctg cag ccc ggg ggt acc gat ccc gag ctc

HIV-1

g gat ce ttttta gg gaa gat ctg gec ttc cca caa ggg gag gcc agg gaa ttt tct tec gag ctc

Ty1

gga tce aat gta tcg aca tct aat aac tct $\operatorname{ccc}$ agc acg gac aac gat tcc atc agt aaa tca act act gaa ccg att caa ttg aac aat aag cac gac ctt cat cttaggc cag aaa ctt act gaa tct aca gta aat cat act aat cat tct gat gat gaa ctc cct gga cac ctc ctt ctc gat tca gga gca tca cga acc ctt ata aga gag ctc

\section{Ty3}

gga tce aga aat aac aac cca tct aga gaa gaa tgt ata aaa aat cgg cta tgc ttc tat tgt aag aaa gag gga cat cgc ctg aac gaa tgt aga. gca cgt aag gegagtt cta acc gat ctt gaa ctc gaa tca aaa gac caa caa act cet ttt atc aaa acc tta cca att gta cac tat atc gcc atc ccc gag atg gac at acc gag ctc

FIGURE 1. Sequences of the pYDL series of frameshift reporter vectors. (Top panel) A schematic of the dual-luciferase cassette subcloned into $\mathrm{p} 416 \mathrm{ADH}$ is pictured. Transcription of the dual-luciferase cDNA is driven by the $A D H 1$ promoter and proper $3^{\prime}$ end formation is facilitated by the CYC1 terminator. (Bottom panels) The sequences of the programmed frameshift signals subcloned into the pYDL series of plasmids are shown. The control plasmid (pYOLcontrol) contains polylinker sequence only and firefly luciferase is in the same reading frame as Renilla luciferase. The predicted reading frame of the mRNA before and after a programmed frameshift is indicated by the spaces. The viral sequences were subcloned into the Bam HI and Sac I sites of the polylinker region between the Renilla and firefly cDNAs. The heptanucleotide slippery sites for each signal and restriction sites used for cloning are indicated in boldface. in the field, and recommend it for the development of in-

\section{The dual-luciferase reporter mRNAs are transcribed}

The first requirement in the full characterization of a new in reporter assay is to ascertain that the reporter mRNAs are plasmids (Fig. 1) was introduced into yeast cells by transformation, and their encoded products were assayed by RNA and immunoblot methodologies. The presence of firefly luciferasespecific hybridizing mRNA species was readily detected in all of the samples, confirming that the reporter mRNAs were (Fig. 2A) First, the presence of a slower migrating species suggests that transcription of small subset of mRNAs is either initiated slightly upstream from the canonical transcriptional start site or ended downstream of the CYC1 transcription termination species appear constant with regard to the major species, their presence should have no real impact on the assay itself. The second point arises from the observation that the steady-state abundances of the frameshift reporter mRNAs appear to be significantly less than that of the 0 -frame control (compare using the nonspecific band as a loading control). Because the dual-luciferase frameshift reporter mRNAs contain in-frame termination codons, they could be substrates for the nonsense-mediated mRNA decay pathway (NMD; for review, see Gonzalez et al. 2001). Similar experiments using monocistronic lacZ-based reporters did not reveal substantial differences in the steady-state abundances of nonsense-containing frameshift reporter lacZ mRNAs as compared with 0-frame controls (Harger et al. 2001; Kinzy et al. 2002), suggesting that these mRNAs are suboptimal for use in eukaryotic expression systems. It has not escaped our notice that the potential sensitivity of the new dual-luciferase reporters to NMD makes them amenable to addressing the controversial question of whether or not the products of the UPF genes are truly involved in programmed -1 ribosomal frameshifting (Bidou et al. 2000; Dinman et al. 2000; Stahl et al. 2000). Studies de- 
A

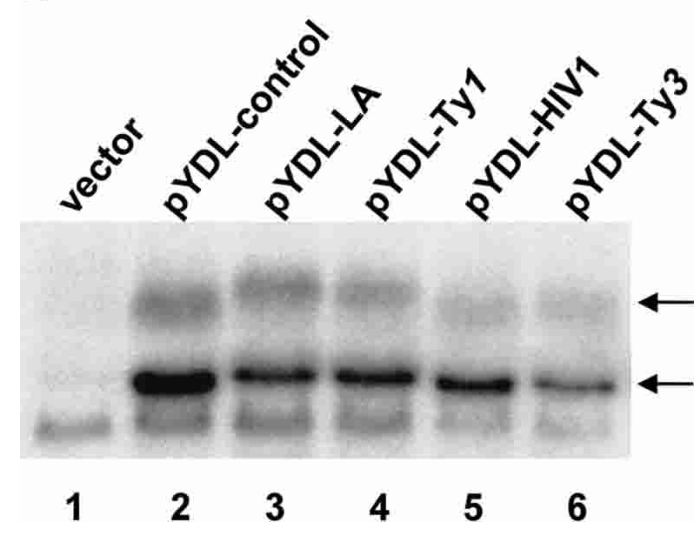

B

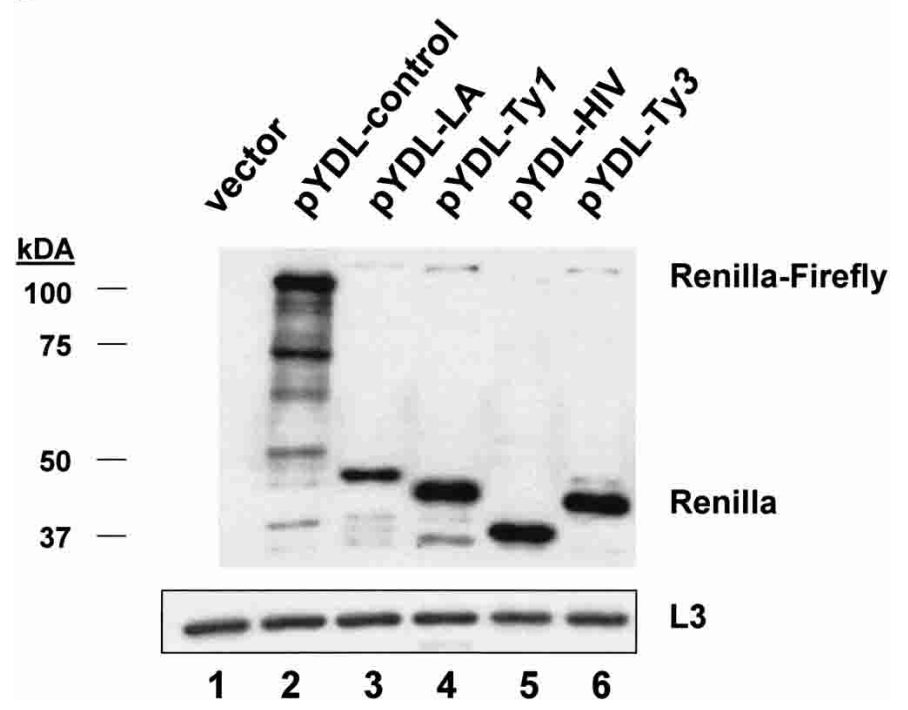

FIGURE 2. The dual-luciferase mRNAs are transcribed and translated. (A) Total RNA was extracted from JD987 cells harboring either an empty vector or the pYDL series of plasmids. RNA $(10 \mu \mathrm{g})$ from each strain was separated through a $1 \%$ agarose-formaldehyde gel, transferred to a nylon membrane and hybridized with an antisense riboprobe specific to the $3^{\prime}$ end of the firefly luciferase cDNA. Two species were observed that were proportional in size and intensity and specific to cells expressing the dual-luciferase cassettes (cf. lanes 1 and 2-6). (B) The same cells were disrupted by glass bead lysis and total protein from a clarified lysate $(5 \mu \mathrm{g})$ was separated by SDS-PAGE and subjected to immunoblot analysis using anitbodies directed against the Renilla luciferase protein and yeast ribosomal protein L3 (loading control). Immunoreactive products were observed in cells harboring the pYDL plasmids (cf. lanes 1 and 2-6) and corresponded to the predicted size of the non-frameshifted (40-50 kD) and frameshifted $(100-110 \mathrm{kD})$ products.

signed to definitively answer this question are currently under way in our laboratory.

Having ascertained transcription of the dual-luciferase reporter genes in vivo, immunoblot analyses of yeast cell lysates were affected to confirm the synthesis of their encoded protein products using an anti-Renilla luciferase monoclonal antibody. The results demonstrate the presence of immunoreactive proteins of the expected apparent molecular weights (Fig. 2B). Specifically in the frameshift reporter constructs, though the majority of the immunoreactive species corresponded to Renilla luciferase plus construct-specific carboxy-terminal extensions, the higher molecular weight Renilla-firefly luciferase fusion proteins were visualized as minor species. The decreased mobilities of the frameshift fusion proteins relative to the 0 -frame encoded fusion protein confirms that these are the anticipated programmed ribosomal frameshift products. Reprobing of the stripped blot with an anti-ribosomal protein L3 monoclonal antibody reveals that equal amounts of protein were present in each sample, further demonstrating the specificity of the immunoreactive species.

\section{Linearity and practical working range of the assay}

To examine the sensitivity and linear range of the assay system, the activities of Renilla and firefly luciferase prod- ucts were determined in the context of the 0 -frame control and the HIV-1 programmed -1 ribosomal frameshift reporter plasmids through an $\sim 4$-order of magnitude range of concentrations of crude yeast cell lysates. The results demonstrate that the assay is essentially linear throughout the entire range, though there is a slight drop-off of linearity in the frameshift reporter luciferase (pYDL-HIV) at the lower end of the assayed range (Fig. 3A). Most importantly, because yeast lysates prepared as described in Materials and Methods typically produce yields of $\sim 0.1-1.0 \mu \mathrm{g} / \mu \mathrm{L}$, little to no dilution of lysates is necessary under the assay conditions used.

Programmed ribosomal frameshifting efficiencies are calculated by dividing the firefly/Renilla luciferase activity ratio determined from lysates of cells expressing the frameshift reporter vector by the same ratio determined from samples derived from cells expressing the 0 -frame control plasmid. To determine the applicability of the assay system to a wide variety of programmed ribosomal frameshift signals, the programmed ribosomal frameshifting efficiencies were determined using lysates of cells in which the frameshift reporter vectors contained the L-A or HIV-1 programmed -1 , or the Ty 1 or Ty 3 programmed +1 ribosomal frameshift signals (Fig. 3B). Using this system, the PRF efficiencies of L-A, HIV-1, and Ty1 were all very similar, suggesting perhaps a common pathway or mechanism for virus particle assembly. 
A

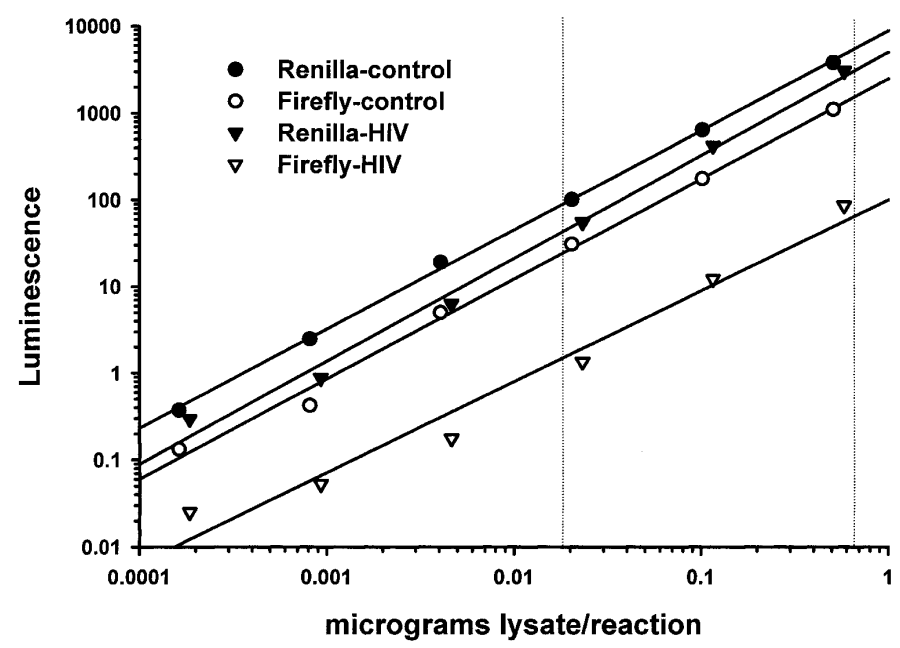

B

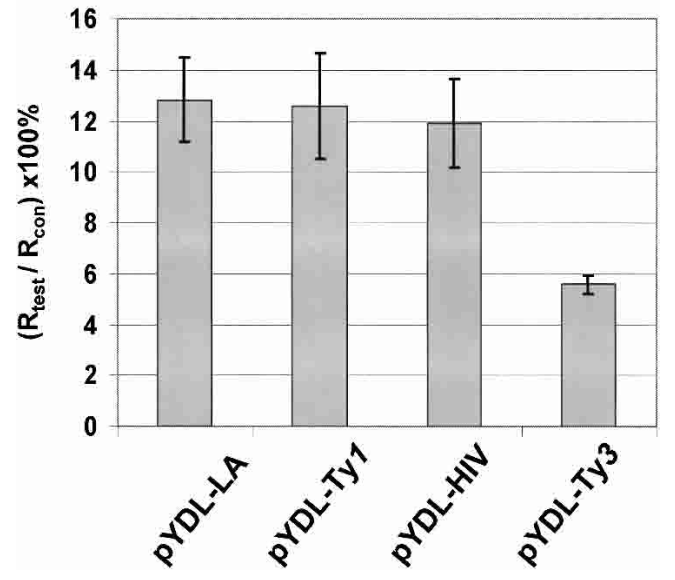

FIGURE 3. Estimation of recoding efficiency. (A) To gauge the linearity and optimal range of the assay system, JD987 cells harboring either pYDL-control or pYDL-HIV were grown to mid-log phase in selective media and crude lysates were obtained as described in Materials and Methods. The protein concentrations of the lysates were determined by the Bradford assay. Several 1:5 dilutions of the lysates were prepared in lysis buffer and $5 \mu \mathrm{L}$ aliquots were assayed in triplicate using the Dual-Luciferase System (Promega) reagents. Averaged luminescence values for the firefly and Renilla reactions for each dilution were plotted on a log-log graph. Linear regression lines for each series shows that the assay is linear over several orders of magnitude. Vertical lines indicate the optimal working range of the assay. (B) JD987 cells harboring the entire series of plasmids were cultured as above and crude lysates were prepared as described in the Materials and Methods within the working range of protein lysate concentration described above. The method of Grentzmann (Grentzmann et al. 1998) was used to calculate frameshifting efficiencies. Briefly, the activity ratio derived from lysates expressing the test cassettes was divided by the activity ratio of the control reporter and multiplied by $100 \%$. All assays were performed in triplicate $3-5$ times.

\section{Advantages of using the yeast in vivo dual-luciferase programmed ribosomal frameshifting reporter system}

The assay system described here presents numerous improvements on those that are currently used in the field. First, the bicistronic nature of the reporter better resembles the genomic organization of viruses as compared with assay systems relying on monocistronic reporters. Second, measurement of reporter enzyme activities is more sensitive and reliable than densitometric methods used to quantify radiolabeled or immunoreactive frameshift products. Third, the use of luciferase reporters circumvents issues of $\beta$-galactosidase toxicity to yeast cells. Fourth, a major advantage of bicistronic reporter systems is that they are internally controlled. This eliminates variability that may arise as a consequence of indirect methods of normalization required for monocistronic assay systems, for example, normalization using optical density/absorbance methods, or for total protein concentrations among different samples. The internally controlled system also controls for changes that may arise as a consequence of differences in mRNA abundance between reporters, and for those that may arise as a consequence of differential rates of translation between test and control reporters. Lastly, the broad linear range of the assay, and the fact that both enzymatic activities can be measured in a single tube make this both ergonomically and economically attractive with regard to potential high-throughput screening applications.

\section{MATERIALS AND METHODS}

\section{Genetic methods and plasmid construction}

Escherichia coli strain DH5 $\alpha$ was used to amplify plasmids, and $E$. coli transformations were performed using the standard calcium chloride method as described previously (Sambrook et al. 1989). Yeast cells were transformed using the alkali cation method (Ito et al. 1983). Yeast cells were grown on YPAD and synthetic complete medium lacking uracil (H-) (Wickner and Leibowitz 1976). To construct the yeast-based dual-luciferase reporter plasmids, the dual-luciferase cDNA was first amplified using the High Fidelity Plus PCR System (Roche) using p2Luci and other variations of this plasmid (see below; Grentzmann et al. 1998) as a template. The primers y2Luc-F 5'-CCCCACTAGTCCACCAATGACTTCGAA AG-3' and y2LucR 5'-CCCCCTCGAGCACGTGTTACAATTTGG ACT-3' containing the Spe I and Xho I restriction sites respectively, were used for amplification by PCR. Reaction products were restricted with Spe I and Xho I, separated through $0.8 \%$ agarose $1 \times$ TAE, and gel purified (Qiagen). These fragments and similarly digested and purified p416 ADH (CEN6 ARSH4 URA3; Mumberg et al. 1995) were ligated and amplified in E. coli DH5 $\alpha$ to create pYDL-control (pJD375). In vivo, transcription of the dual-luciferase reporter mRNA is driven by the $A D H 1$ promoter and proper $3^{\prime}$ end formation is facilitated by the $C Y C 1$ terminator contained within the plasmid. To construct the yeast HIV-1 test reporter, pJD187.WT (Dinman et al. 2002) was used as a template with the same primers and conditions as above. To construct the yeast L-A, Ty1 and Ty 3 test reporters, the respective frameshift signals were first subcloned into the polylinker region of p2Luci (Grentzmann 
et al. 1998). The L-A signal was amplified by PCR from plasmid pF8 (Dinman et al. 1991) with Taq polymerase (Qiagen) using the primers LA2LucF 5'-CCCCGGATCCACTTCTAGGATCAATGC GGG-3' and LA2LucR 5'-CCCCGAGCTCGGGATCGGTACCCC CGG-3' containing the Bam HI and Sac I sites respectively. PCR products were restricted with Bam HI and Sac I, gel purified (as above), and ligated into similarly digested p2Luci to create pJD206. The Ty1 frameshift signal was amplified from pJEFF1105 (Boeke et al. 1988) with the primers 5TY1LUC 5'-CCCCGGATC CAATGTATCGACATCTAATAACTCT-3' and 3TY1LUC 5'-CCC CGAGCTCTCTTATAAGGGTTCGTGATGC-3'. The Ty3 frameshift signal was amplified from pAGE191 with the primers 5TY3LUC 5' -CCCCGGATCCAGAAATAACAACCCATCTAGAG AA-3' and 3TY3LUC 5'-CCCCGAGCTCGGTATTGTCCATCTC GGGGAT-3'. PCR products were digested and ligated as above into p2Luci to create pJD207 and pJD208 respectively. pJD206, 207, and 208 were used as templates to amplify dual-luciferase cassettes containing recoding signals for subsequent insertion into p416 ADH as described above to create pYDL-LA, pYDL-TY1, and pYDL-TY3 (pJD376, 377, 379) respectively. The sequence of all plasmids was confirmed by automated dye-terminator sequencing.

\section{RNA analysis}

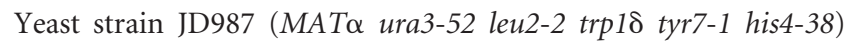
harboring the pYDL series of plasmids were grown in H-Uracil liquid media $(10 \mathrm{~mL})$ to an O.D. ${ }_{595 \mathrm{~nm}}$ of 0.7 . Yeast were harvested by centrifugation, washed once in $\mathrm{dH}_{2} \mathrm{O}$, and resuspended in 0.6 mL Smash \& Grab Buffer (1\%SDS, 2\% Triton X-100, $100 \mathrm{mM}$ $\mathrm{NaCl}, 10 \mathrm{mM}$ Tris $\mathrm{pH} 8.0,1 \mathrm{mM}$ EDTA) along with an equal volume of acid-phenol/chloroform 5:1 (Ambion). Half-millimeter glass beads (0.3 g) (BioSpec) were added and cells were agitated continuously in a vortex mixer for one minute. The aqueous phase was re-extracted with acid phenol/chloroform 5:1 and once more with phenol/chloroform/IAA 25:24:1. RNA was ethanol precipitated and $10 \mu \mathrm{g}$ was separated on $1 \%$ agarose-formaldehyde. After capillary transfer and UV-crosslinking to a nylon membrane (BioRad), immobilized nucleic acids were hybridized with an antisense riboprobe $\left(\alpha\left[{ }^{32} \mathrm{P}\right]\right.$ rCTP-labeled) specific to the $3^{\prime}$ end of the firefly luciferase gene. Dual-luciferase mRNAs were visualized by phosphorimaging (Molecular Dynamics).

\section{Immunoblot analysis}

Yeast cells harboring the pYDL series of plasmids were grown to an O.D. ${ }_{595 \mathrm{~nm}}$ of 0.7 in H-Uracil liquid media $(5 \mathrm{~mL})$. Cells were harvested by centrifugation, washed once with $1 \mathrm{~mL}$ of $1 \times \mathrm{PBS} \mathrm{pH}$ 7.4, $1 \mathrm{mM}$ PMSF, and resuspended in $250 \mu \mathrm{L}$ of the same buffer. Glass beads $(0.3 \mathrm{~g})$ were added and yeast cells were agitated continuously in a vortex mixer at $4^{\circ} \mathrm{C}$ for $3 \mathrm{~min}$. Lysates were clarified by centrifugation and protein concentrations were determined by the Bradford method (BioRad). Five micrograms of the crude lysates were separated by SDS-PAGE and proteins were transferred to a nitrocellulose membrane for immunoblot analysis. After electrotransfer, the membrane was washed three times in MilliQ $\mathrm{H}_{2} \mathrm{O}$ and then blocked for $1 \mathrm{~h}$ in $1 \times \mathrm{PBS} \mathrm{pH} 7.4,5 \%$ Nonfat dry milk. The membrane was incubated for $1.5 \mathrm{~h}$ in $20 \mathrm{~mL}$ of a 1:2000 dilution of a mouse anti-Renilla IgG mAb (clone \#2Q2159, US Biological $)$ in $1 \times \operatorname{PBST}(0.5 \%$ Tween-20) $\mathrm{pH} 7.4,2 \%$ nonfat dry milk, and then washed for 45 min with multiple changes in $1 \times$ PBST. The membrane was then incubated with a 1:2000 dilution of an HRP-conjugated anti-mouse secondary antibody (Amersham) as above and then washed for $15 \mathrm{~min}$ as above. Immunoreactive species were detected using chemiluminescent reagents (Perkin Elmer). The membrane was stripped in $0.1 \mathrm{M}$ glycine $\mathrm{pH}$ 3.0 for $30 \mathrm{~min}$ and subsequently reblocked as above. The membrane was subsequently probed for ribosomal protein L3 using an anti-yeast L3 monoclonal antibody.

\section{Dual-luciferase assays}

Typically, JD987 cells harboring the pYDL series of plasmids were grown in H-uracil liquid media $(1 \mathrm{~mL})$ to an O.D. $595 \mathrm{~nm}$ of 0.7 . Cells were harvested by centrifugation and washed once with $1 \mathrm{~mL}$ of ice cold lysis buffer (1× PBS pH 7.4, 1 mM PMSF) and then resuspended in $0.3 \mathrm{~mL}$ of the same buffer. Cells suspensions were lysed with glass beads and processed as described above. Typically the concentrations of crude lysates were between 0.1 and $1.0 \mathrm{mg} /$ $\mathrm{mL}$ as determined by the Bradford method (BioRad). Luciferase activities were determined using $5 \mu \mathrm{L}$ of lysate/sample using the Dual-Luciferase Assay System (Promega) and a TD 20/20 luminometer (Turner Designs). Frameshift efficiencies were calculated using the method described previously (Grentzmann et al. 1998). The firefly/Renilla activity ratio generated from the control reporter (pYDL-control) was divided into that from the frameshift reporters (pYDL-LA, HIV, TY1, and TY3) and multiplied by $100 \%$ to obtain frameshift efficiencies for each recoding signal. All assays were performed in triplicate at least three times. To determine the linearity of the assay, several 1:5 serial dilutions of yeast lysates from JD987 harboring the pYDL-control and pYDL-HIV were prepared in lysis buffer and luciferase activities were determined as described above.

\section{ACKNOWLEDGMENTS}

We thank Dr. Abram Gabriel for providing us with pAGE191 and Dr. Jonathan Warner for generously providing us with high-quality anti-yeast L3 monoclonal antibodies. We also thank Kristi Muldoon-Jacobs for technical assistance. This work was supported by a grant to J.D.D. from the National Institutes of Health (R01 GM58859).

The publication costs of this article were defrayed in part by payment of page charges. This article must therefore be hereby marked "advertisement" in accordance with 18 USC section 1734 solely to indicate this fact.

Received October 23, 2002; accepted May 22, 2003.

\section{REFERENCES}

Beckett, G.J. and Tatum, E.L. 1941. Genetic control of biochemical reactions in Neurospora. Proc. Natl. Adac. Sci. 27: 499-506.

Bidou, L., Stahl, G., Hatin, I., Namy, O., Rousset, J.P., and Farabaugh, P.J. 2000. Nonsense-mediated decay mutants do not affect programmed -1 frameshifting. RNA 6: 952-961.

Boeke, J.D., Xu, H., and Fink, G.R. 1988. A general method for the chromosomal amplification of genes into yeast. Science 239: 280 282.

Dinman, J.D., Icho, T., and Wickner, R.B. 1991. A -1 ribosomal frame- 
shift in a double-stranded RNA virus forms a Gag-pol fusion protein. Proc. Natl. Acad. Sci. 88: 174-178.

Dinman, J.D., Ruiz-Echevarria, M.J., and Peltz, S.W. 1998. Translating old drugs into new treatments: Identifying compounds that modulate programmed -1 ribosomal frameshifting and function as potential antiviral agents. Trends Biotech. 16: 190-196.

Dinman, J.D., Ruiz-Echevarria, M.J., Wang, W., and Peltz, S.W. 2000. The case for the involvement of the Upf3p in programmed -1 ribosomal frameshifting. RNA 6: 1685-1686.

Dinman, J.D., Richter, S., Plant, E.P., Taylor, R.C., Hammell, A.B., and Rana, T.M. 2002. The frameshift signal of HIV-1 involves a potential intramolecular triplex RNA structure. Proc. Natl. Acad. Sci. 99: 5331-5336.

Farabaugh, P.J. 1996. Programmed translational frameshifting. Microbiol. Rev. 60: 103-134.

Garrod, A.E. 1902. The incidence of alkaptonuria: A study in clinical individuality. Lancet 2: 1616-1620.

Gesteland, R.F. and Atkins, J.F. 1996. Recoding: Dynamic reprogramming of translation. Annu. Rev. Biochem. 65: 741-768.

Gonzalez, C.I., Bhattacharya, A., Wang, W., and Peltz, S.W. 2001. Nonsense-mediated mRNA decay in Saccharomyces cerevisiae. Gene 274: 15-25.

Grentzmann, G., Ingram, J.A., Kelly, P.J., Gesteland, R.F., and Atkins, J.F. 1998. A dual-luciferase reporter system for studying recoding signals. RNA 4: 479-486.

Harger, J.W., Meskauskas, A., Nielsen, N., Justice, M.C., and Dinman J.D. 2001. Ty1 retrotransposition and programmed +1 ribosomal frameshifting require the integrity of the protein synthetic translocation step. Virology 286: 216-224.

Harger, J.W., Meskauskas, A., and Dinman, J.D. 2002. An "integrated model" of programmed ribosomal frameshifting and post-transcriptional surveillance. Trends Biol. Sci. 27: 448-454.

Ito, H., Fukuda, Y., Murata, K., and Kimura, A. 1983. Transformation of intact yeast cells treated with alkali cations. J. Bacteriol. 153: $163-168$.

Kinzy, T.G., Harger, J.W., Carr-Schmid, A., Kwon, J., Shastry, M., Justice, M.C., and Dinman, J.D. 2002. New targets for antivirals: The ribosomal A-site and the factors that interact with it. Virology 300: $60-70$.

Mumberg, D., Muller, R., and Funk, M. 1995. Yeast vectors for the controlled expression of heterologous proteins in different genetic backgrounds. Gene 156: 119-122.

Sambrook, J., Fritsch, E.F., and Maniatis, T. 1989. Molecular cloning, a laboratory manual (eds. C. Nolan et al.). Cold Spring Harbor Laboratory Press, Cold Spring Harbor, NY.

Stahl, G., Bidou, L., Rousset, J.-P., and Cassan, M. 1995. Versatile vectors to study recoding: Conservation of rules between yeast and mammalian cells. Nucleic Acids Res. 23: 1557-1560.

Stahl, G., Bidou, L., Hatin, I., Namy, O., Rousset, J.P., and Farabaugh, P. 2000. The case against the involvement of the NMD proteins in programmed frameshifting. RNA 6: 1687-1688.

Wickner, R.B. and Leibowitz, M.J. 1976. Two chromosomal genes required for killing expression in killer strains of Saccharomyces cerevisiae. Genetics 82: 429-442. 

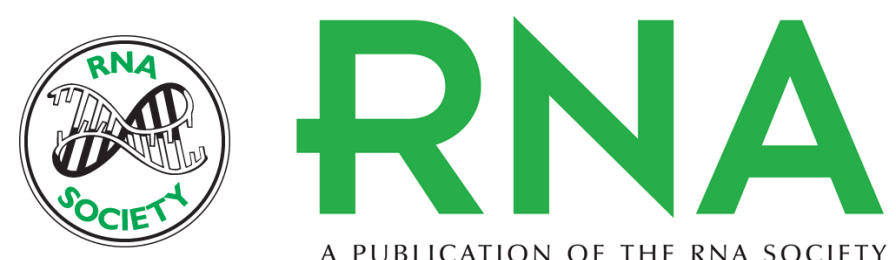

A PUBLICATION OF THE RNA SOCIETY

\section{An in vivo dual-luciferase assay system for studying translational recoding in the yeast Saccharomyces cerevisiae}

JASON W. HARGER and JONATHAN D. DINMAN

RNA 2003 9: 1019-1024

References This article cites 19 articles, 9 of which can be accessed free at: http://rnajournal.cshlp.org/content/9/8/1019.full.html\#ref-list-1

\section{License}

Email Alerting Service

Receive free email alerts when new articles cite this article - sign up in the box at the top right corner of the article or click here. 